\title{
Electrokinetics Technology to Improve Acidizing of Carbonate Reservoir Rocks
}

\author{
Sanghee Shin ${ }^{1 *}$, George V. Chilingar ${ }^{1}$, Mohammed Haroun ${ }^{2}$, Kenneth Wittle ${ }^{3}$, Najmedin Meshkati ${ }^{1}$, \\ Sibel Pamukcu ${ }^{4}$, Jaehyeung Jeoung ${ }^{5}$, Hobon $\mathrm{Koo}^{5}$
} ${ }^{1}$ Viterbi School of Engineering, University of Southern California, Los Angeles, USA; ${ }^{2}$ Petroleum Institute, Abu Dhabi, UAE;
${ }^{3}$ Electropetroleum Inc., Villanova, USA; ${ }^{4}$ Lehigh University, Bethlehem, USA; ${ }^{5}$ Geotechnical Engineering Research Division, Korea
Institute of Construction Technology, Goyang City, South Korea.

Email: *sangshin@usc.edu

Received January $24^{\text {th }}, 2013$; revised February $27^{\text {th }}, 2013$; accepted March $29^{\text {th }}, 2013$

Copyright (C) 2013 Sanghee Shin et al. This is an open access article distributed under the Creative Commons Attribution License, which permits unrestricted use, distribution, and reproduction in any medium, provided the original work is properly cited.

\begin{abstract}
A novel technology (electrokinetics) is proposed to improve acidizing operations, i.e., increase the penetration distance. The acid dissolves the carbonates (limestones/dolomites), enlarging the pores and increasing the width of pre-existing fractures. This gives rise to an increase in permeability. The principal acid commonly used is hydrochloric $(\mathrm{HCl})$, which is pumped through tubing. Aqueous solutions of hydrochloric acid (usually 15\%) are pumped into the carbonate formations to enlarge the pores and pre-existing fractures. Without application of D.C. current, the penetration distance is usually very short, especially in tight rocks. However, the penetration distance of acid is very short. By applying D.C. current, one can drive the acid for long distances into the formation being acidized.
\end{abstract}

Keywords: Acidizing; Electorkinetics; Carbonate Rocks; Penetration Distance; Stimulation

\section{Introduction}

Stimulation of carbonate reservoirs is achieved chiefly by acidizing treatments [1-6]. Acids may be injected into pores and pre-existing fractures or at hydraulic fracturing rates depending upon the results desired. The acid dissolves the carbonates (limestones/dolomites), enlarging the pores and increasing the width of pre-existing fractures. This gives rise to an increase in permeability. The principal acid commonly used is hydrochloric $(\mathrm{HCl})$, which is pumped through tubing.

In the case of acidizing through pre-existing fractures, with increasing width of fractures, 1) the specific surface area decreases, 2) the spending time increases, and 3) the penetration distance increases.

The main problem in acidizing is the fact that the radial distance the acid will penetrate until being spent is short, especially in tight carbonates.

As shown in Equation (1), in order to increase $r_{a}$, either $t$ or $q_{i}$ should be increased. As the experimental data obtained by the writers indicate, the injection rate $q_{i}$, can be increased considerably by application of D.C. current (electrokinetic effect, Figure 1) [2-4]. The application of

${ }^{*}$ Corresponding author.
D.C. electrokinetics in Abu Dhabi carbonate reservoir rocks has been proven to be very promising by the authors [7].

\section{Discussion}

On assuming a homogeneous formation and that the

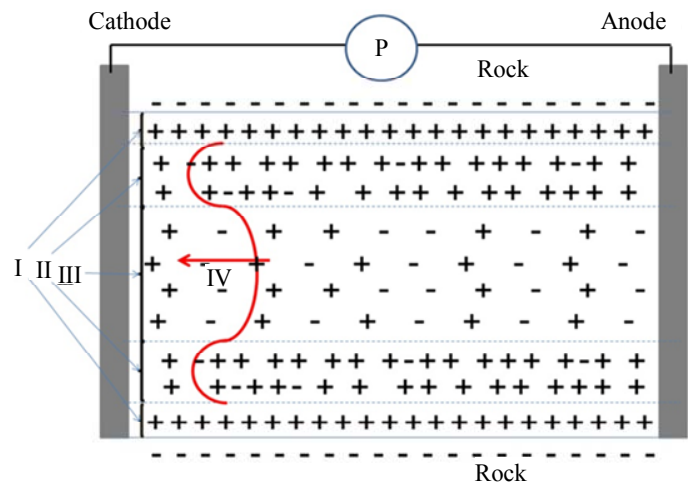

Figure 1. Schematic diagram of electrokinetic double layer (I: Immobile Double Layer, II: Mobile Double Layer, III: Free Water, IV: Velocity Profile) as envisioned by the authors. Solid curved line-velocity profile in a capillary. $\mathbf{P}=$ D.C. current power supply. Rock is negatively charged [8]. 
volume of acid injected is equal to the pore volume invaded $\left[q_{i} t=\pi \Phi h\left(r_{a}^{2}-r_{w}^{2}\right)\right]$, the radial distance the acid will penetrate until being spent, $r_{a}(\mathrm{ft})$, is equal to;

$$
r_{a}=\sqrt{\frac{q_{i} t}{\pi \phi h}+r_{w}^{2}}
$$

where:

$q_{\mathrm{i}}=$ acid injection rate $(\mathrm{bbl} / \mathrm{min}) ; t=$ spending time (sec); $\phi=$ fractional porosity; $h=$ formation thickness (ft); and $r_{w}=$ wellbore radius (ft).

In the case of uniform penetration of acid, the reaction rate declines uniformly with decreasing acid concentration. The weight of carbonate dissolved per increment of distance penetrated declines uniformly until the acid is completely spent. With stronger acid, the spending time decreases.

In the case of matrix acidizing, with enlargement of pores, 1) the specific surface area decreases, 2) the velocity decreases, 3) spending time increases, and 4) the penetration distance increases.

When the imposed electrical potential gradient $(E)$ is in the same direction as the pressure drop, the flow rate increases:

$$
q_{t}=\frac{A k \Delta p}{\mu L}+\frac{A k_{e} E}{\mu L}
$$

where:

$q_{t}=$ total volumetric rate of flow (electrokinetic plus hydrodynamic); $k=$ hydrodynamic permeability; $A=$ cross-sectional area; $L=$ length of porous media; $\Delta p=$ pressure drop; $\mu=$ viscosity; $k_{e}=\frac{D \xi}{4 \pi F}$, is the electrokinetic permeability; $F=$ formation resistivity factor (Archie's); $D=$ dielectric constant; and $\xi=$ zeta potential.

If Equation (3) is presented in a dimensionless form by normalizing the flow rates and, thus, eliminating the viscosity, area and length terms:

$$
\frac{q_{t}}{q_{i}}=1+\frac{k_{e} E}{k \Delta p}
$$

and

$$
\frac{\left(q_{t}-q_{i}\right)}{q_{i}}=\frac{k_{e} E}{k \Delta p}
$$

where:

$q_{i}=$ initial hydrodynamic stabilized flow rate.

Equation (4) shows that an increase in the flow rate is dependent upon the zeta potential, dielectric constant, brine concentration, Darcy permeability, and pressure drop. This equation also suggests that as the hydrodynamic permeability decreases, the percent increase in flow rate due to electrokinetics will become more significant. It

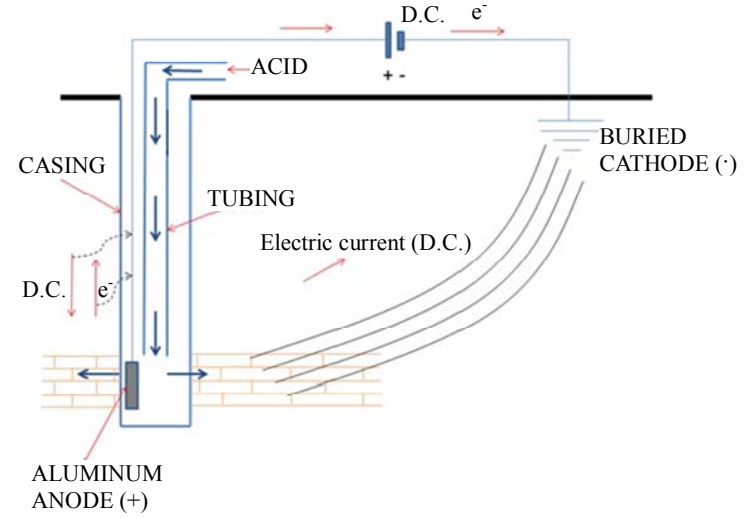

Figure 2. Electrodes arrangement for acidizing operation.

should be remembered, however, that viscosity decreases with increase in temperature due to the application of an electrical potential. In tight formations, $k_{e}$ may exceed $k$ considerably. The feasibility of application of D.C. current in carbonate reservoir rocks with acids was demonstrated by Haroun et al. (2009) [7].

\section{Deployment System for Enhanced Acidzing}

As shown in Figure 2, using electrokinetics, it is necessary to deploy an anode in the well adjacent to the formation being acidized, and a nearby cathode either at the surface or in the adjoining well. The electrokinetic flow will occur from the anode towards the cathode, and thereby acidizing the target formation, enabling the acid to move faster and deeper into the formation in a guided fashion [9]. The two electrodes (anode and cathode) must be connected by cables to the Direct Current power supply located on the surface. The acid must be injected with corrosion inhibitors into the formation; however, the aluminum anode may also serve as sacrificial anode.

\section{Conclusion}

The proposed technology (electrokinetics) to improve acidizing operations is indeed a breakthrough. By applying D.C. current, the volumetric rate of flow increases, which, in turn, increases the penetration distance of the acid before it is being spent. Without application of D.C. current, the penetration distance is usually very short, especially in tight rocks.

\section{REFERENCES}

[1] A. R. Herdrickson, "Stimulation of Carbonate Reservoirs," In: G. V. Chilingar, R. W. Mannon and H. H. Rieke, Eds., Oil and Gas Production from Carbonate Rocks, Elsevier, 1972, pp. 309-339.

[2] G. V. Chilingar, J. E. Davis, H. J. Farhanghi, L. G. Adam- 
son and S. Sawabini, "Possible Use of Direct Electrical Current for Augmenting Reservoir Energy during Petroleum Production," Compass of Sigma Gamma Epsilon, Vol. 45, No. 4, 1968, pp. 272-285.

[3] G. V. Chilingar, A. El-Nassir and R. G. Stevens, "Effect of Direct Electrical Current on Permeability of Sandstone Cores," Journal of Petroleum Technology, Vol. 22, No. 7, 1970, pp. 830-836. doi:10.2118/2332-PA

[4] G. V. Chilingar, R. W. Mannon and H. H Rieke, "Oil and Gas Production from Carbonate Rocks," Elsevier, New York, 1972.

[5] G. V. Chilingar, S. V. Mazzullo and H. H. Rieke, "Carbonate Reservoir Characterization," A Geologic-Engineering Analysis, Part I, Elsevier, Amsterdam, 1992.

[6] B. C. Craft, W. R. Holden and E. E. Graves Jr., "Well De- sign, Drilling and Production," Prentice Hall, Englewood Cliffs, 1992.

[7] M. R. Haroun, G. V. Chilingar, S. Pamukcu, J. K. Wittle, H. A. Belhaj and M. N. Al Bloushi, "Optimizing Electroosmotic Flow Potential for Electrically Enhanced Oil Recovery (EEORTM)," 2009.

[8] S. H. Shin, "Electroremediation of Offshore Muds Contaminated with Heavy Metals," Ph.D. Dissertation, University of Southern California, 2011.

[9] J. K. Wittle, D. G. Hill and G. V. Chilingar, "Direct Electric Current Oil Recovery (EEOR)-A New Approach to Enhancing Oil Production," Energy Sources, Part A (Recovery, Utilization, and Environmental Effects), Vol. 33, No. 9, 2011, pp. 805-822. 